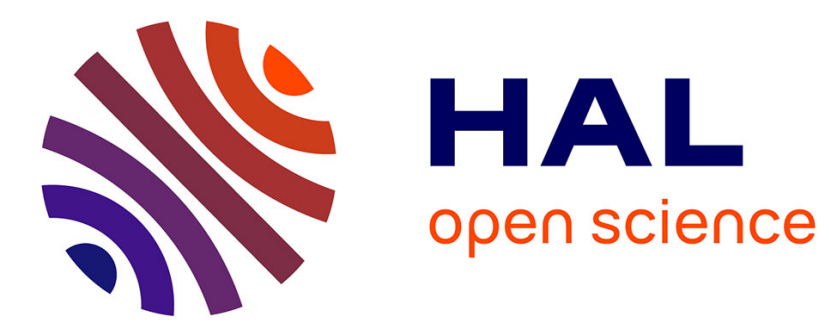

\title{
Impact of Expressive Wrinkles on Perception of a Virtual Character's Facial Expressions of Emotions
}

Matthieu Courgeon, Stéphanie Buisine, Jean-Claude Martin

\section{To cite this version:}

Matthieu Courgeon, Stéphanie Buisine, Jean-Claude Martin. Impact of Expressive Wrinkles on Perception of a Virtual Character's Facial Expressions of Emotions. 9th International Conference, IVA 2009, Sep 2009, Netherlands. pp.201-214, 10.1007/978-3-642-04380-2_24 . hal-00783596

\section{HAL Id: hal-00783596 https://hal.science/hal-00783596}

Submitted on 1 Feb 2013

HAL is a multi-disciplinary open access archive for the deposit and dissemination of scientific research documents, whether they are published or not. The documents may come from teaching and research institutions in France or abroad, or from public or private research centers.
L'archive ouverte pluridisciplinaire HAL, est destinée au dépôt et à la diffusion de documents scientifiques de niveau recherche, publiés ou non, émanant des établissements d'enseignement et de recherche français ou étrangers, des laboratoires publics ou privés. 


\title{
Impact of Expressive Wrinkles on Perception of a Virtual Character's Facial Expressions of Emotions
}

\author{
Matthieu Courgeon ${ }^{1}$, Stéphanie Buisine ${ }^{2}$, Jean-Claude Martin ${ }^{1}$ \\ ${ }^{1}$ LIMSI-CNRS, B.P. 133, 91403 Orsay, France \\ \{courgeon, martin\}@limsi.fr \\ ${ }^{2}$ Arts et Métiers ParisTech, LCPI, 151 bd Hôpital, 75013 Paris, France \\ stephanie.buisine@paris.ensam.fr
}

\begin{abstract}
Facial animation has reached a high level of photorealism. Skin is rendered with grain and translucency, wrinkles are accurate and dynamic. These recent visual improvements are not fully tested for their contribution to the perceived expressiveness of virtual characters. This paper presents a perceptual study assessing the impact of different rendering modes of expressive wrinkles on users' perception of facial expressions of basic and complex emotions. Our results suggest that realistic wrinkles increase agent's expressivity and user's preference, but not the recognition of emotion categories. This study was conducted using our real time facial animation platform that is designed for perceptive evaluations of affective interaction.
\end{abstract}

Keywords: Facial animation, Evaluation of virtual agents, Affective interaction, Advanced 3D modeling and animation technologies

\section{Introduction}

Facial expressions are "rapid signals produced by the movements of the facial muscles, resulting in temporary changes in facial appearance, shifts in location and shape of the facial features, and temporary wrinkles" [12]. Since this early definition, facial expressions have been extensively studied. Expressive virtual characters based on Ekman's work are now widely used. However, most virtual agents do not display wrinkles. Indeed, most of them use the MPEG-4 animation system, which does not integrate wrinkles. Thus, few perceptive studies on expressive virtual faces have assessed the role of expressive wrinkles on the perception of emotions. Does their presence vs. absence play a role in emotion decoding? Does expressive wrinkles depth influence emotions perception? Does realism influence user's preference?

This paper presents a perceptive study on the influence of different levels of expressive wrinkles rendering on subjects' perception of emotion. We considered not only basic emotions, but we also explored more complex emotions. A video presenting this study is available on the web ${ }^{1}$.

In section 2, we present some related works. We review theories of emotions and studies on the perception of wrinkles in psychology distinguishing permanent and

${ }^{1}$ URL : http://www.limsi.fr/Individu/courgeon/static/IVA09/ 
expressive wrinkles. We also provide an overview of virtual character animation and expressive wrinkles generation. Section 3 presents MARC, our interactive facial animation system, extending the MPEG-4 model to display expressive wrinkles and enabling different wrinkle rendering modes. Section 4 presents our experiment, the results of which are discussed in section 5. Section 6 concludes this paper and presents future directions.

\section{Related work}

An emotion can be seen as an episode of interrelated, synchronized changes in five components in response to an event of major significance to the organism [31]. These five components are: the cognitive processing, the subjective feeling, the action tendencies, the physiological changes, and the motor expression.

Ekman suggests different characteristics which distinguish basic emotions from one another and from other affective phenomena [10]. He lists distinctive clues for the facial expressions of Surprise, Fear, Disgust, Anger, Happiness, and Sadness [12]. Each of the basic emotions is not seen as a single affective state but rather as a family of related states. Several researchers (Tomkins, Izard, Plutchik and Ekman) consider different lists of fundamental emotions. For example Izard's list includes Contempt, Interest, and Guilt. Five emotions are nevertheless common to the lists proposed by these four researchers (Anger, Disgust, Joy, Fear and Surprise). Baron-Cohen proposes a more general and detailed list of 416 mental states including for example Fascination [13]. Although less literature is available about the facial expressions of these mental states (some of which are called complex emotions by Baron-Cohen), the MindReading database includes 6 audiovisual acted expressions for each of these mental states [13].

In this paper we wish to address the influence of wrinkles on emotional expression or perception. Following Ekman's distinction [9], we consider separately wrinkles as rapid signs vehicles of emotional expression (i.e. when they are temporarily produced by the activity of the facial muscles) and slow sign vehicles (i.e. permanent wrinkles emerging over the life span). Outside Ekman's descriptions of wrinkles in expressions of basic emotions [12], temporary wrinkles are sometimes mentioned anecdotally, e.g. crow's feet typically involved in Duchenne smile [11]. However, we failed to find in psychology literature any formal attempt to model the influence of these temporary wrinkles on emotional expression.

Besides, the role of permanent wrinkles is sometimes discussed in emotional aging research and we briefly review this literature. The dominant theory in this field states that emotional processes (experience and expression) should be submitted to the general decline associated to aging. Self-report surveys from elderly people tend to confirm a decrease in emotional experience, although this decline may arise in different ways for positive and negative affects [14, 25]. Physiological reactions to emotional experience also decline with age [18]. An alternate theory suggests that these phenomena may be due to a greater emotional control rather than a decline [3, $14,21]$, but the consequences on emotional expression are the same. Indeed, several experimental data show that elderly people's facial expressions are harder to decode, 
be they voluntarily elicited [18, 22] or produced by mood-induction procedures [23]. However, opposite results were also reported, showing no difference in emotional expressiveness between older and younger adults [16, 21]. Results from Borod et al. [3] might provide a nice hypothesis to account for such discrepancy as well as for the aforementioned theoretical assumptions. These authors instructed young, middle-aged and old women to produce negative, positive emotions, and neutral facial expressions. These posed expressions were subsequently evaluated by independent judges, and the results highlight two opposite phenomena: on the one hand, the expressions of older posers proved to be less accurate and decoded with less confidence than those of younger posers, which is consistent with either a decline of emotional expression over age, or a greater emotional control with more masking and blends. On the other hand, the neutral poses of older subjects were rated as more intense than those of younger people, which can be due to age-related morphological changes in the face, i.e. permanent wrinkles and folds [3]. Furthermore, these permanent wrinkles remaining visible on neutral expressions can convey personality information [22], e.g. anger dominance in a personality trait tends to leave a permanent imprint on the face.

Following this set of results, we can hypothesize that wrinkles should increase facial expressiveness, although in humans this effect is sometimes compensated by a decline or a greater control in emotional expression, possibly resulting in a global absence of difference between young and old peoples' level of expressiveness, or in confusing blends in older people. Therefore, in virtual characters systems, wrinkles are expected to enhance expressiveness, as far as no decline, control or interference process is simulated.

Since the early $70 \mathrm{~s}$, research in computer graphics tries to simulate the human face, perceived as a powerful communication tool for human-computer interaction. Parke animated a virtual human face with a short number of parameters [28], creating an animation by linear interpolation of key expressions and using a simple face representation. This method is still used in several systems by interpolating between key expressions or keypoints' position [30]. For example, the MPEG-4 model [27] is widely used for expressive virtual agents [26, 30]. However, interpolation based models have limitations, e.g. the non-compliance to face anatomical constrains. Several approaches were proposed to model multiple layers of the face (e.g. bones, muscles, and skin), e.g. Anatomic models such as Waters' muscles models [37] and Terzopoulos' model [32]. However, they require more computational time than parametric models. Most of these models are not real-time.

In the early $90 \mathrm{~s}$, the increasing performance of computers and the emergence of programmable GPUs (Graphic Processing Unit) gave a new impetus to facial animation. Viaud generated procedural expressive wrinkles [34]. Wu simulated skin elasticity properties [39]. Anatomical models also benefited of these new hardware performances, and real-time anatomically accurate models have appeared.

Facial animation addresses another issue: credibility. Synchronized animations all over the face and linear interpolation of facial expressions are perceived as unnatural motions [29]. Pasquariello et al. [30] divide the face into 8 areas, and involve a local area animation at different speed rates.

On a real human face, expressions create expressive wrinkles, with varying intensity and depth, depending on age and morphology. However, simple models like 
MPEG-4 do not offer a systematic way to simulate wrinkles. Several techniques have been proposed to simulate such effects, and they are not specific to facial animation, e.g. cloth simulation [15]. Wrinkle generation can be divided in two approaches. Firstly, predefined wrinkles, manually edited or captured, and triggered during animation $[15,20]$. This technique requires one or several wrinkle patterns for each model. Larbourlette et al. [17] used a compression detection algorithm applied to mesh triangles to trigger predefined wrinkles. The wrinkles progressively appear as the mesh is compressed.

The second main approach is the generative method, e. g. physical computation of muscles and skin elasticity. It does not need predefined wrinkle patterns. This approach is generally much more complex, and requires more computational time. However, the resulting wrinkles are generated automatically, without manual edition $[4,38]$. Some physical models are specifically developed for wrinkles generation [38]. In contrast, some models generate wrinkles as a side effect of their anatomically based facial animation system. Several generative models have been proposed, based on length preservation, energy functions [36], or mass-spring systems [40].

As generative models require more computation time, some use the GPU to generate wrinkles [19]. The method is similar to Larboulette's work [17], but the wrinkle pattern is dynamically generated in the GPU. However, this approach uses a large part of GPU capacities that recent facial animation systems need for realistic skin rendering. Combining procedural and generative models, Decaudin et al. [7] propose an hybrid approach for clothes simulation, defining manually folding lines, and generating wrinkles automatically.

Several methods exist to create facial animation and expressive wrinkles. Evaluations of these methods are often limited to technical criteria, such as frame rate, computational complexity, or the fuzzy concept of "realism". However, in a context in which virtual faces are used to convey an affective content, perceptive evaluations are required. As argued by Deng [8], human perception is one the most effective measuring tool for expressivity of a virtual agent. Some perceptive studies provide recommendations for conception and design of virtual agents. For example, studies on the "uncanny valley" [35], assess the interaction between visual and behavioral realism of an agent. The "persona effect"[33] reports how the presence of an agent modifies user's perception of a task and can improve his performance and/or preference. But no such study was conducted on expressive wrinkles. Some studies on complex emotions show that they can modify user's perception. For example, as argued by Becker-Asano [2], agents expressing both basic and complex emotions are perceived to be older than agents expressing only basic emotions.

To summarize, few expressive virtual agents are displaying sophisticated wrinkles. Most of them use the MPEG-4 system, which does not include wrinkles generation. Thus, no detailed studies have been conducted to assess the impact of different features of wrinkles, e.g. depth, visual and dynamic realism. However, the technology to generate such expressive wrinkles does exist. In this paper, we present a perceptual study led with our facial animation platform, extending the MPEG-4 to display dynamic expressive wrinkles. Our study assesses the impact of the presence vs. 
absence of expressive wrinkles, and the impact of wrinkles realism of the recognition of emotions.

\section{MARC: A facial animation platform for expressive wrinkles}

MARC (Multimodal Affective and Reactive Character)[5] is designed for real-time affective interaction. It relies on GPU programming to render detailed face models and realistic skin lighting. This technique enables a more realistic rendering than most of the existing interactive virtual agents. Our animation system extends the MPEG-4 model [27] and uses additional techniques to render expressive wrinkles. As in the MPEG-4 animation system, key expressions are predefined as a set of keypoints displacements, and our system achieves real-time animation by blending several key expressions. Thus, we can create complex facial expressions from predefined ones. We developed a dedicated offline 3D edition software, enabling direct 3D edition of keypoints position, displacement, and influence on the facial mesh. This software enables manual edition of wrinkles directly on the face. All outputs are compatible with our real-time rendering engine. During online animation, live blends of key expressions are performed. In addition, several automatic features are computed from the dynamic facial expression, e.g. expressive wrinkles activation and eyelids position. Visual realism is achieved using recent graphic computing techniques for skin rendering [6]. We compute real-time simulation of skin translucency (BSSRDF) and cast shadows.

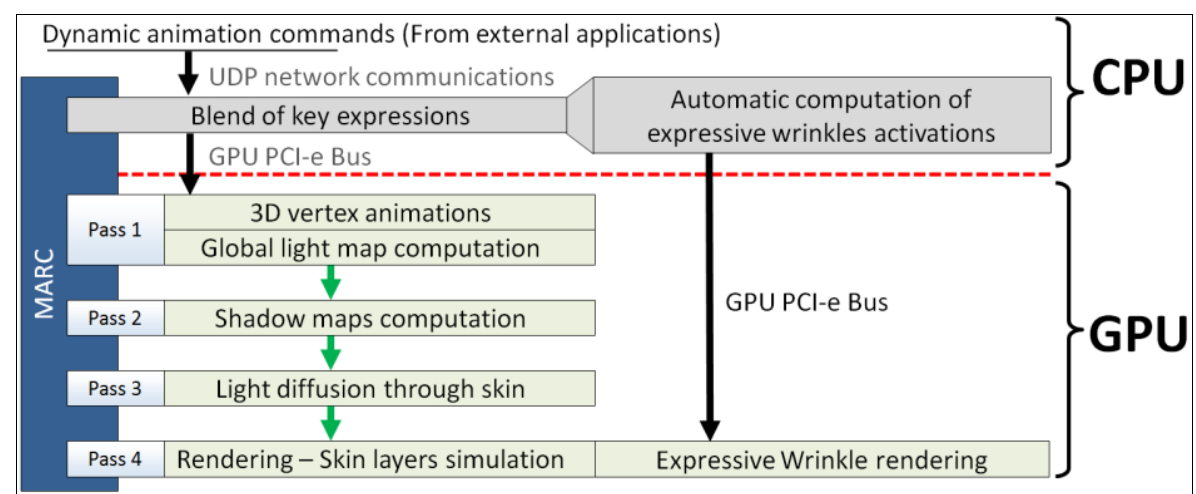

Fig. 1. Rendering and animation pipeline of our animation system.

Fig. 1 shows the multi-pass rendering and animation pipeline. Pass \#1 performs "per vertex" animation and computes a light map. This map is used in pass \#2 to generate shadow maps. Pass \#3 simulates light diffusion through the skin to generate final illumination maps. Finally, pass \#4 uses all resulting information to render a realistic face and generate wrinkles.

Dynamic facial expression is achieved by blending key expressions. Expressive wrinkles are then triggered from facial deformation. Triggering is based on an adaptation of clothes wrinkling [17] to MPEG-4 facial animation. Instead of computing global mesh compression to deduce wrinkles visibility, we compute the 
compression of the keypoints' structure to deduce wrinkles visibility. These compression rules are designed to match the different expressive wrinkles described in Ekman's descriptions of facial expressions [12].

Fig. 2 shows the different compression rules. Joy triggers crow's feet wrinkles $(\mathrm{C}$, and $\mathrm{F}$ axes) and naso-labial folds ( $\mathrm{A}$ and $\mathrm{B}$ axes). Anger triggers vertical lines between the brows (H axis).

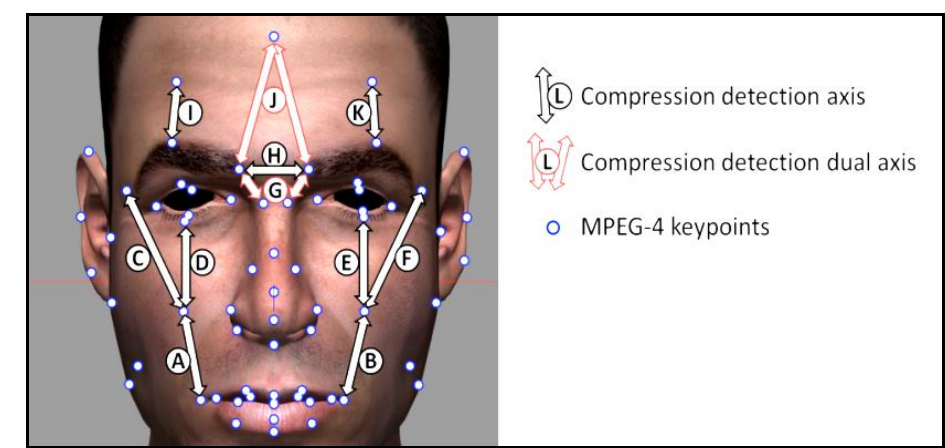

Fig. 2. Compression detection axis for wrinkles triggering

From the compression rules, we obtain wrinkles visibility percentages that we use in the GPU (Pass \#4) to draw wrinkles with variable intensity. Our platform enables different modes of rendering. The "No-Wrinkles" mode does not render wrinkles (e.g. only the movements of eyebrows, lips etc. are displayed; the texture remains the same). The "Realistic-Wrinkles" mode renders smooth bumpy wrinkles. The "Symbolic-Wrinkles" mode renders black lines instead of realistic wrinkling effect, generating non realistic but visible wrinkles. Finally, the "Wrinkles-Only" mode is displaying realistic wrinkles without any actual movement on the face (e.g. the face shape remains unchanged, but its texture seems to fold). Fig. 3 shows the Anger expression with all wrinkle modes.

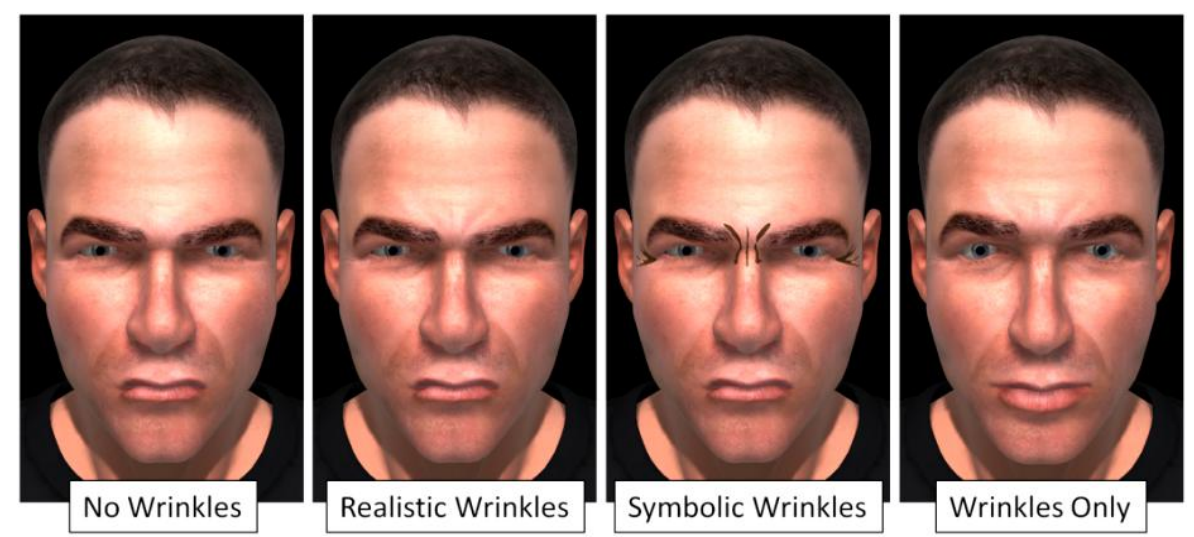

Fig. 3. Anger expression using the 4 wrinkle modes. 


\section{Experiment}

Our study aims at assessing the impact of the presence vs. absence of expressive wrinkles, and the impact of expressive wrinkles realism. Facial expressions of basic emotions have been specified in detail including expressive wrinkles [12]. Nevertheless, it has been shown that a larger set of affective states than 6 basic emotions exists $[1,24]$. Thus, our goal in this experiment is to study wrinkles effects on a larger set of affect, including some complex emotions (cf. below).

Our first hypothesis is that basic emotions will be better recognized than complex affective states as they were proved to be universally recognized. We also hypothesize that different wrinkles rendering models will show differences in recognition rates. Finally, as we will use different intensities, we suppose that expressions with higher intensities will be better recognized. We defined the full intensity of an expression as the facial movements' threshold over which we perceived the expression as too exaggerated. The low intensity expression is defined as a proportional reduction of full-intensity facial movements. Finally, we hypothesize that differences between wrinkle rendering modes will be less significant with lower emotion intensities.

\subsection{Experimental setup}

Participants. 32 subjects (10 females, 22 males), aged from 16 to 40 (25 years old on average, $\mathrm{SD}=4.6$ ) participated in the experiment.

Material. The expression of 8 emotions were designed: 4 basic emotions (Joy, Anger, Fear, and Surprise) and 4 complex emotions (Interest, Contempt, Guilt, and Fascination). To limit the number of stimuli, only emotions with positive Arousal [24] were selected. We selected a basic emotion and a complex emotion on each quarter of the Pleasure/Dominance [24] space. A facial expression of each basic emotions was defined using Ekman's description [12]. Facial expressions of selected complex emotions (Interest, Contempt, Guilt, and Fascination) were inspired by the MindReading database [1] where each mental state is acted by six actors (we extracted facial expressions features, e.g. brows movements, appearing in at least half of the videos). Emotion categories were selected within the intersection of Russell and Mehrabian [24] set of affective states and Baron-Cohen mental states [1]. This selection method was used in order to have for each emotion its location in the PAD space, and a video corpus of its facial expression.

Each selected emotion was expressed using the 4 wrinkles models (No wrinkle, Realistic wrinkles, Symbolic wrinkles, Wrinkles only) with 2 different intensities. Each animation started with a neutral face, then expressed progressively the current emotion, sustained it for 4 seconds, and got back to a neutral face. Animations were rendered in real-time using $2 \mathrm{nVidia}$ 8800GT graphic cards (SLI), and displayed on a 24 " screen with a $1920 \times 1200$ pixels resolution. A video describing these stimuli was submitted to IVA 2009 along with the current paper.

Procedure. Subjects were invited to provide some personal information (age, gender, occupation, etc.). The experiment was divided in 2 phases. The first phase consisted in watching successively 64 short animations displaying a facial expression. For each animation, subjects had to select a single label in a set of 16 emotional 
descriptors. Fig. 4 shows the 16 descriptors we selected as possible answers. 8 were the displayed emotions. 4 adjectives were selected by neutralizing the Pleasure axis or the Dominance axis, and 4 adjectives selected with a negative Arousal.

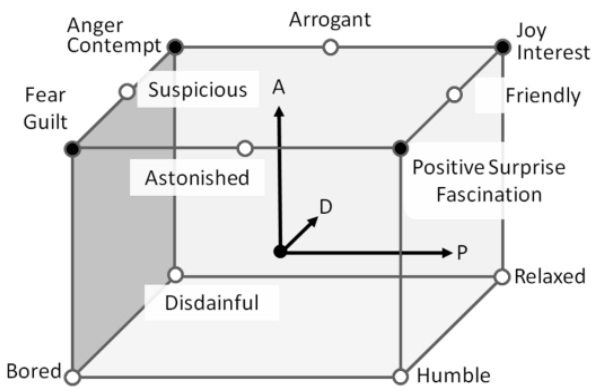

Fig 4. Selected emotions (black dots) and descriptors (white dots) in the PAD space.

4 animations served as a training session, with no time limit. From the $5^{\text {th }}$ animation, subjects had only 30 seconds to choose a label. We set up this timeout procedure in order to ensure a relative spontaneity of answers and to limit the total duration of the experiment. The presentation order of the 64 stimuli was randomized across the subjects' sample. Participants were allowed to take a short break between 2 animations by clicking on a pause button.

In the second phase, for each emotion, participants were shown static images of the emotional expression at the maximum intensity and using the 4 graphical rendering modes side by side in a randomized order (emotion and rendering). Participants had to rank these renderings modes according to their expressivity level for each emotion. They also had to choose their favorite rendering for each emotion. The whole experiment lasted about 30 to 40 minutes by subject.

Data collected. The recognition of emotion was collected as a binary variable (right answer $=1$, wrong answer $=0)$. The ranking of expressivity $\left(1^{\text {st }}\right.$ rank representing the stimulus perceived as most expressive) was converted into expressivity scores ( $1^{\text {st }}$ rank became a 3-point score of expressivity) and the preferences were collected as a binary variable (favorite rendering $=1$, other $=0$ ).

\subsection{Results}

Recognition performance, Expressivity scores and Preference scores were analyzed by means of ANOVAs with Gender as between-subject variable, Emotional category, Graphical rendering and Intensity as within-subject variables. Fisher's LSD was used for post-hoc pair-wise comparisons. All the analyses were performed with SPSS.

Recognition performance. Among the 64 stimuli $\times 32$ users (i.e. 2048 items), there were 56 timeouts, which corresponds to $2.7 \%$ of data. They were analyzed as wrong recognition answers. The average answer time over the whole sample of items was 15.6 seconds $(\mathrm{SD}=1.99)$. The global recognition score was $26.6 \%$, the chance level being at $6.25 \%$ (drawing lots out of 16 emotional labels). The main effect of Emotional category proved to be significant $(F(7 / 630)=16.24, p<0.001$, see Fig. 5 left panel): the recognition score was higher than the chance level, except for Guilt and 
Fascination (9.3\% and 9.8\%) whose scores were not significantly different from the chance level.
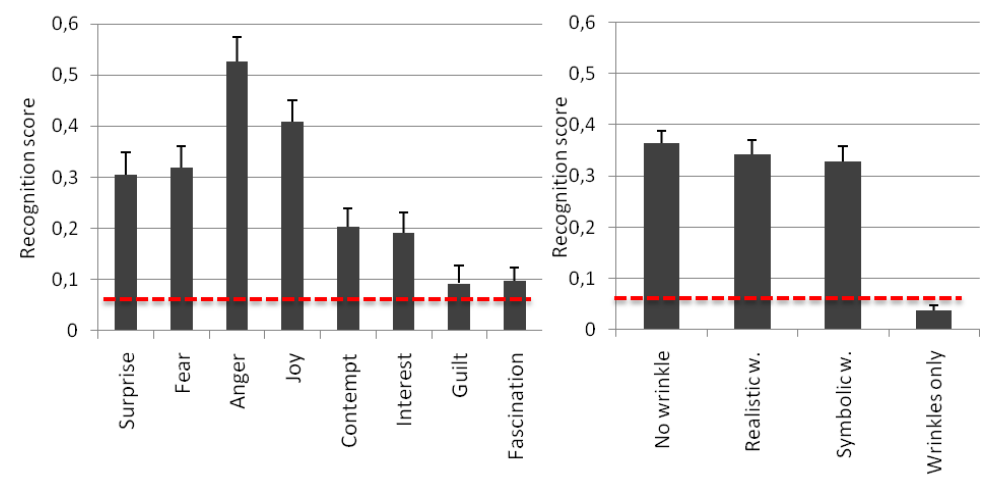

Fig. 5. Global recognition scores for each Emotional category (left panel) and each Graphical rendering (right panel). The dotted horizontal line represents the chance level (0.0625).

Moreover, basic emotions were better recognized (39\%) than complex emotions (14.6\%, p $<0.05)$. Anger was best recognized (52.7\%) among basic emotion (marginally from Joy, $\mathrm{p}=0.059$, and significantly from all other emotions, $\mathrm{p}<0.002$ ).

The main effect of Graphical rendering was also significant $(F(3 / 630)=59.39$, $\mathrm{p}<0.001$, see Fig. 5 right panel): the No-wrinkle, Realistic-wrinkle and Symbolicwrinkle renderings all enabled equivalent recognition scores, significantly higher than the chance level $(34.5 \%, \mathrm{p}<0.001)$. Conversely the recognition score with Wrinklesonly rendering $(3.7 \%)$ was not different from the chance level.

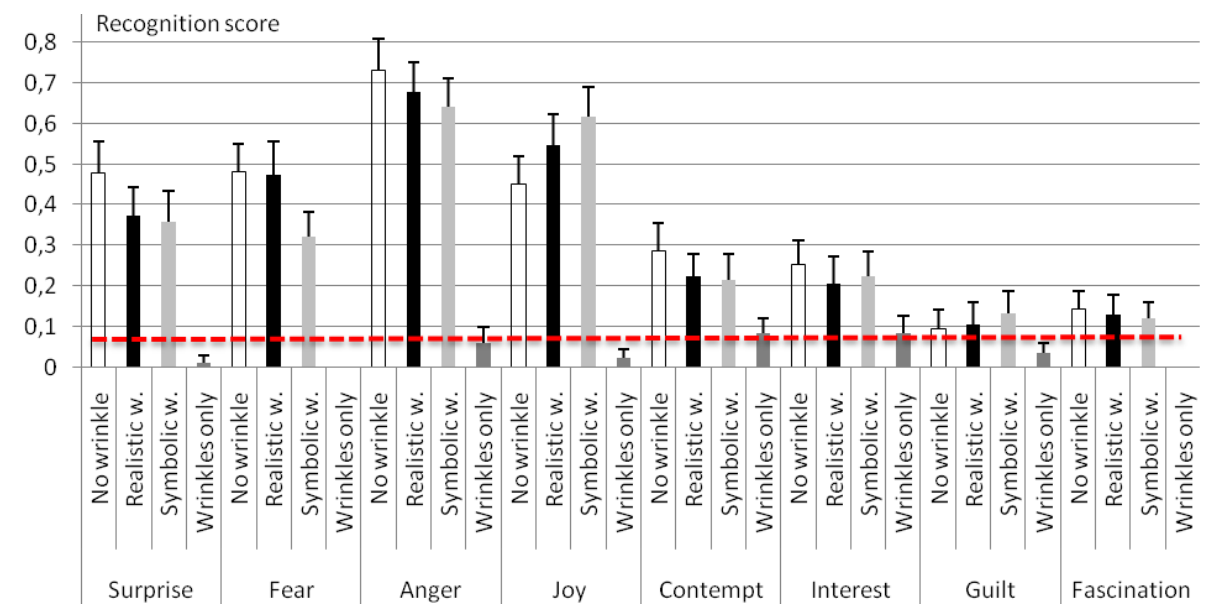

Fig. 6. Recognition scores for each Emotional category $\times$ Graphical rendering combination. The dotted horizontal line represents the chance level (0.625). 
We also observed an Emotional category $\times$ Graphical rendering interaction $(\mathrm{F}(21 / 630)=5.27, \mathrm{p}<0.001$ see Fig. 6$)$, showing that the previous pattern (No-wrinkle, Realistic-wrinkle, and Symbolic-wrinkle renderings equivalent and better than Wrinkles-only rendering) was not true for all emotional categories. For Interest and Guilt there was no effect of graphical rendering. Besides, for Joy the Symbolic rendering gave rise to marginally better performance than the No-Wrinkle rendering $(p=0.078)$. Finally, for the recognition of Fear, the Symbolic rendering was associated to a marginally lower performance than No wrinkle $(\mathrm{p}=0.065)$ and Realistic wrinkles $(\mathrm{p}=0.073)$

The stimuli intensity also had a significant influence on the recognition score $(\mathrm{F}(1 / 630)=12.01, \mathrm{p}=0.002)$ : items displayed with high intensity were better recognized $(30 \%)$ than items displayed at medium intensity $(23.7 \%)$. However, an Emotional category $\times$ Intensity interaction $(\mathrm{F}(7 / 630)=2.95, \mathrm{p}=0.006)$ showed that this pattern was true only for Fear $(p<0.001)$ and Anger $(p=0.014)$. For all other emotions, the Intensity of stimuli had no influence on the recognition performance.

Finally, subject's Gender had no influence on the recognition performance.

Expressivity. Regarding the subjective evaluation of Expressivity, the Graphical rendering proved to have a significant main effect $(\mathrm{F}(3 / 630)=97.39, \mathrm{p}<0.001$, see Fig. 7 left panel). The Realistic-wrinkle rendering was rated as significantly more expressive $(2.4 / 3)$ than the 3 other renderings $(\mathrm{p}<0.001)$. The scores of No-wrinkle and Symbolic-wrinkle renderings (1.6 and 1.7) were not significantly different while the Wrinkles-only rendering was significantly the least expressive $(0.2, \mathrm{p}<0.001)$.
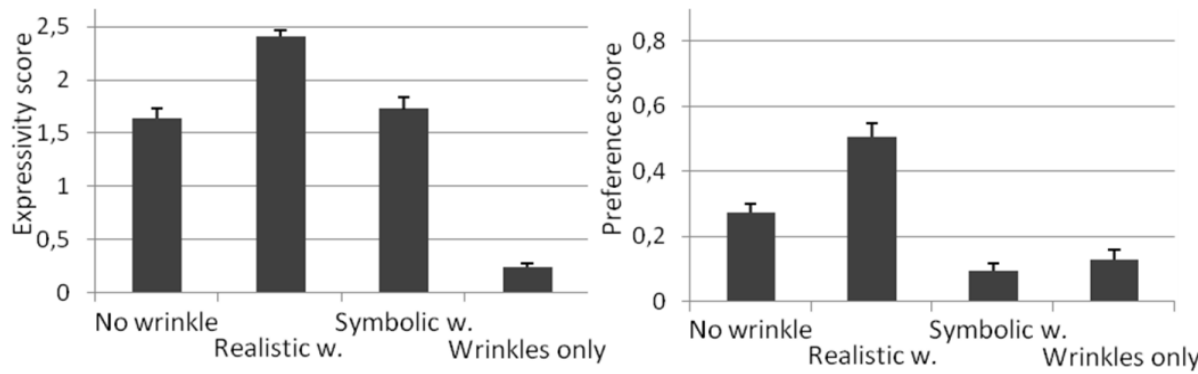

Fig. 7. Expressivity scores (left) and Preference scores (right) for each Graphical rendering.

An Emotional category $\times$ Graphical rendering interaction $(F(21 / 630)=5.96$, $\mathrm{p}<0.001$ ) showed several patterns according to the Emotional category:

- For Surprise, Guilt and Fascination: the main effect is verified (Realistic wrinkles most expressive; No-wrinkle and Symbolic equivalent; Wrinkles-only least expressive).

- For Fear and Anger: Realistic wrinkles most expressive; Symbolic rendering more expressive than No-wrinkle rendering; Wrinkles-only rendering least expressive.

- For Joy and Contempt: Wrinkles-only is still the least expressive rendering, but no significant difference between the 3 other renderings.

- For Interest: Realistic wrinkles most expressive; then No-wrinkle; Symbolic and Wrinkles-only equivalent and least expressive.

Subject's Gender had no significant influence on Expressivity ratings. 
Preferences. The Graphical rendering had a significant main effect on the Preference scores $(\mathrm{F}(3 / 630)=25.80, \mathrm{p}<0.001$, see Fig. 7 right panel $)$. The realistic wrinkles (with a preference score of $0.51 / 1$ ) were preferred to the 3 other renderings $(\mathrm{p}<0.001)$. Then the No-wrinkle rendering $(0.27)$ was preferred to Symbolic wrinkles $(0.09)$ and to Wrinkles-only $(0.13, \mathrm{p}<0.001)$. Symbolic and Wrinkles-only had equivalent preference scores.

An Emotional category $\times$ Graphical rendering interaction $(\mathrm{F}(21 / 630)=2.58$, $\mathrm{p}<0.001$ ) showed 4 preferences patterns according to the Emotional category:

- For Surprise, Fear and Anger: the Realistic rendering is preferred $(p<0.037)$ while the 3 other renderings are not different.

- For Joy, Guilt and Fascination: the No-wrinkle rendering is not significantly different from the Realistic rendering.

- For Interest: The difference between the Wrinkles-Only mode and the Realistic mode is not significant.

- For Contempt: The effect of the graphical rendering is not significant, which means that no rendering was consistently preferred.

\section{Discussion}

Our results provide hints about the validity of our experimental stimuli. The fact that the recognition scores were higher than the chance level tends to validate the design of our animations, except for Guilt and Fascination which were not better recognized than the chance level. Therefore, we will no longer discuss the results about these 2 emotional expressions. When the recognition data about Guilt and Fascination are excluded, the recognition score on the remaining expressions amounts to $32.6 \%$. Such performance level may still seem low, but we would like to underline that only strictly exact answers were taken into account in our analyses and that slight vs. major recognition errors were not distinguished within wrong answers. For example, confusion between Contempt and Arrogance was considered as a wrong answer although people may overlook the difference between the expressions of these emotions. We adopted this conservative rule in order to prevent our data from a ceiling effect and maximize the likelihood of observing differences between our graphical renderings.

The global effect of intensity on recognition scores also tends to validate our stimuli, as high intensities lead to better recognition rates. However, this improvement is only significant for Fear and Anger expressions. Indeed, the recognition of the other emotional expressions is not significantly better with intense stimuli. We hypothesize that our way of manipulating intensity is responsible for this result. High intensity of an emotion may not be expressed only by a wider expression, but using different facial expressions for a single emotion and other modalities, such as head and gaze movements, and skin coloration. Further studies should be conducted to validate this hypothesis.

The main goal of this experiment was to compare several graphical renderings related to wrinkle display. In this respect, the major, original and unexpected result of this 
study is the effect of renderings on the recognition of emotions. The presence of wrinkles did not improve the recognition performance: neither realistic nor symbolic wrinkles seemed to provide more information than the no-wrinkle rendering. Hence the key factor for facial expression recognition appears to be face movements rather than wrinkles, since the absence of movements (wrinkles-only condition) made the recognition performance dramatically fall to the chance level. However, the realisticwrinkle rendering proved to be rated as the most expressive and the favorite one of our subjects. Such result is sufficient to ground the usefulness of including realistic wrinkles into virtual characters design. Regarding expressivity, one may wonder why our participants rated realistic rendering as more expressive although it did not provide effective recognition improvement. We hypothesize that these ratings of expressivity were influenced by users' preferences, which may have led them to pick up this rendering first in the ranking exercise of the experiment.

The 3 interaction effects between emotional categories and graphical renderings (on recognition, expressivity and preference scores) provide us with finer information as to whether some renderings fit more or less to particular emotional expressions. However, when synthesizing the results from the 3 variables, some patterns appear inconsistent or confusing; therefore they cannot be used directly as generic design recommendations. For example, for Joy the symbolic wrinkles tended to improve recognition but had lower preference scores than the realistic and no-wrinkle renderings. For Fear, the symbolic wrinkles were rated as more expressive than the no-wrinkle rendering but tended to disrupt the recognition. For Interest, the wrinklesonly rendering obtained a preference score equivalent to that of realistic wrinkles but was rated as least expressive.

Basic emotions were better recognized than complex ones. This can be explained by the lack of detailed descriptions of facial expressions of complex emotions in the literature. We also hypothesize that definition of these emotions are more subjective. Thus, selecting an adjective for these emotions is a fuzzier task.

\section{Conclusion and future directions}

We presented an experiment using our platform for interactive facial animation. Our goal was to evaluate the impact of expressive wrinkles on user's perception of emotion expressed by a virtual character. The major result of this experiment is that wrinkles simulation did not improve categorical recognition of emotions. However, wrinkles influence user's preferences and improve perceived expressivity of a virtual character. We were unable to provide systematic design recommendations for the conception of virtual face, mainly because of the recognition differences between emotions. However, we cannot conclude that wrinkles have no effects on categorical recognition. For this experiment, we only used two kinds of wrinkle simulation, only one "realistic", and none of them were generated by a physical system. Thus, other types of wrinkle simulation may have an effect on categorical recognition. Similar experiments on anatomically accurate wrinkles simulation have to be conducted.

Moreover, our study was limited in several ways. A single emotion was displayed at a time, whereas wrinkles might also have a role to play in the perception of blends 
of emotions. We only used a male virtual face. We did not consider static wrinkles due to aging. Finally, an emotion was always expressed with one single expression.

To summarize, our study suggests that expressive wrinkles should be realistically rendered on a virtual face, as it improves user's preference and agent's expressivity. Virtual agents are now used in a wide range of applications. It is thus important to take into account such results in the design of expressive virtual agents.

\section{References}

1. Baron-Cohen, S.: Mind Reading: The Interactive Guide to Emotions. Jessica Kingsley Publishers (2007).

2. Becker-Asano, C., Wachsmuth, I. (2008). Affect Simulation with Primary and Secondary Emotions, Proceedings of the 8th international conference on Intelligent Virtual Agents. Tokyo, Japan: Springer-Verlag.

3. Borod, J., Yecker, S., Brickman, A., Moreno, C., Sliwinski, M., Foldi, N., Alpert, M., Welkowitz, J.: Changes in posed facial expression of emotion across the adult life span. Experimental Aging Research, 30 (2004) 305-331.

4. Bridson, R., Marino, S., Fedkiw, R. (2005). Simulation of clothing with folds and wrinkles, ACM SIGGRAPH 2005 Los Angeles, California: ACM.

5. Courgeon, M., Martin, J.-C., Jacquemin, C. (2008). User's Gestural Exploration Of Diffirent Virtual Agents' Expressive Profiles, 7th Int. Conf. on Autonomous Agents and Multiagent Systems (AAMAS'2008). Estoril - Portugal.

6. D'Eon, E., Luebke, D.: Advanced techniques for realistic real-time skin rendering. In nguyen (Ed.), GPU Gems 3, Addison Wesley, (2007) 293-348.

7. Decaudin, P., Thomaszewski, B., Cani, M.-P. (2005). Virtual garments based on geometric features of fabric buckling: INRIA.

8. Deng, Z., Ma, X.: Perceptually Guided Expressive Facial Animation. SCA'08: Proc. of SIGGRAPH/EG SCA(2008) 67-76.

9. Ekman, P.: Facial signs: Facts, fantasies, and possibilities. In T. Sebok (Ed.), Sight, sound and sense, Indiana University Press, Bloomington (1978) 124-156.

10. Ekman, P.: Basic emotions. In T. Dalgleish \& M.J. Power (Eds.), Handbook of Cognition \& Emotion, John Wiley, New York (1999) 301-320.

11. Ekman, P., Davidson, R.J., Friesen, W.V.: The Duchenne Smile: Emotional Expression and Brain Physiology II. Journal of Persotutlity and Social Psychology, 50(2), (1990) 342-353.

12. Ekman, P., Friesen, W.V.: Unmasking the face. A guide to recognizing emotions from facial clues. Englewood Cliffs, New Jersey: P-H. , (1975).

13. Golan, O., Baron-Cohen, S., Hill, J.: The Cambridge Mindreading (CAM) Face-Voice Battery: Testing Complex Emotion Recognition in Adults with and without Asperger Syndrome. Journal of Autism and Developmental Disorders, 36(2), (2006).

14. Gross, J.J., Carstensen, L.L., Pasupathi, M., Tsai, J., Götestam-Skorpen, C., Hsu, A.Y.C.: Emotion and aging: Experience, expression and control. Psychology and Aging, 12, (1997) 590-599.

15. Hadap, S., Bangerter, E., Volino, P., Magnenat-Thalmann, N. (1999). Animating wrinkles on clothes, Proceedings of the conference on Visualization '99: celebrating ten years. San Francisco, California, United States: IEEE Computer Society Press.

16. Kunz, M., Mylius, V., Schepelmann, K., Lautenbacher, S.: Impact of age on the facial expression of pain. Journal of Psychosomatic Research, 64, (2008) 311-318.

17. Larboulette, C., Cani, M.-P. (2004). Real-Time Dynamic Wrinkles, Computer Graphics International. 
18. Levenson, R.W., Carstensen, L.L., Friesen, W.V., Ekman, P.: Emotion, physiology, and expression in old age. Psychology and Aging, 6, (1991) 28-35.

19. Loviscach, J.: Wrinkling coarse meshes on the GPU. CGF 25, (2006) 467--476.

20. Maddock, S., Edge, J., Sanchez, M.: Movement realism in computer facial animation. 19th British HCI Group Annual Conference, Workshop on Human-animated Characters Interaction, (2005).

21. Magai, C., Consedine, N.S., Krivoshekova, Y.S., Kudadjie-Gyamfi, E., McPherson, R.: Emotion experience and expression across the adult life span: Insights from a multimodal assessment study. Psychology and Aging, 21, (2006) 303-317.

22. Malatesta, C.Z., Fiore, M.J., Messina, J.J.: Affect, personality, and facial expressive characteristics of older people. Psychology and Aging, 2, (1987) 64-69.

23. Malatesta, C.Z., Izard, C.E., Culver, C., Nicolich, M.: Emotion communication skills in young, middle-aged, and older women. Psychology and Aging, 2, (1987) 193-203.

24. Mehrabian, J.A.R.a.A.: Evidence for a three-factor theory of emotions. Journal of Research on Personality, (1977) 273-294.

25. Mroczek, D.K.: Age and emotion in adulthood. Current Directions in Psychological Science, 10, (2001) 87-90.

26. Niewiadomski, R., Ochs, M., Pelachaud, C.: Expressions of Empathy in ECAs Lecture Notes in Computer Science, Volume 5208/2008(Intelligent Virtual Agents), (2008) 37-44.

27. Pandzic, I., Forchheimer, R.: MPEG-4 Facial Animation: The Standard, Implementation and Applications. John Wiley I\&amp; Sons, Inc. (2003).

28. Parke, F.I.: A parametric model for human faces. Univ. of Utah (1974).

29. Parke, F.I.: Parameterized Models for Facial Animation. IEEE Comput. Graph. Appl., 2(9), (1982) 61-68.

30. Pasquariello, S., Pelachaud, C.: Greta: A Simple Facial Animation Engine. 6th Online world conf. on soft computing in industrial applications, (2001).

31. Scherer, K.R.: Emotion. In M.H.W. Stroebe (Ed.), Introduction to Social Psychology: A European perspective, Blackwell, Oxford (2000) 151-191.

32. Terzopoulos, D., Waters, K.: Analysis and Synthesis of Facial Image Sequences Using Physical and Anatomical Models. IEEE Trans. Pattern Anal. Mach. Intell., 15(6), (1993) 569-579.

33. Van Mulken, S., André, E., Müller, J. (1998). The Persona Effect: How Substantial Is It?, Proceedings of HCI on People and Computers XIII: Springer-Verlag.

34. Viaud, M.-L., Yahia, H. (1992). Facial animation with wrinkles.

35. Walters, M.L., Syrdal, D.S., Dautenhahn, K., te Boekhorst, R., Koay, K.L.: Avoiding the Uncanny Valley. Robot Appearance, Personality and Consistency of Behavior in an Attention-Seeking Home Scenario for a Robot Companion. Journal of Autonomous Robots, 24(2), (2008) 159-178.

36. Wang, Y., Wang, C.C.L., Yuen, M.M.F.: Fast energy-based surface wrinkle modeling. Computers \& Graphics, 30(1), (2006) 111-125.

37. Waters, K. (1987). A muscle model for animation three-dimensional facial expression, Proceedings of the 14th annual conference on Computer graphics and interactive techniques: ACM.

38. Wu, Y., Magnenat Thalmann, N., Thalmann, D.: A dynamic wrinkle model in facial animation and skin ageing. Journal of Visualization and Computer Animation, 6(4), (1995) 195-205.

39. Wu, Y., Thalmann, N.M., Kalra, P.: Simulation of static and dynamic wrinkles of skin. Proceedings of Computer Animation '96(1996) 90 - 97.

40. Zhang, Y., Prakash, E., Sung, E.: Real-time physically-based facial expression animation using mass-spring system. Computer Graphics International 2001, (2001). 